\title{
New Turán densities for 3-graphs
}

\author{
Rahil Baber \\ 613 Greenford Road, \\ London, UK. \\ rahilbaber@hotmail.com
}

\author{
John Talbot* \\ Department of Mathematics \\ UCL, London, UK. \\ j.talbot@ucl.ac.uk
}

Submitted: Nov 1, 2011; Accepted: May 18, 2012; Published: May 31, 2012

Mathematics Subject Classifications: 05D05

\begin{abstract}
If $\mathcal{F}$ is a family of graphs then the Turán density of $\mathcal{F}$ is determined by the minimum chromatic number of the members of $\mathcal{F}$.

The situation for Turán densities of 3-graphs is far more complex and still very unclear. Our aim in this paper is to present new exact Turán densities for individual and finite families of 3-graphs, in many cases we are also able to give corresponding stability results. As well as providing new examples of individual 3-graphs with Turán densities equal to $2 / 9,4 / 9,5 / 9$ and $3 / 4$ we also give examples of irrational Turán densities for finite families of 3-graphs, disproving a conjecture of Chung and Graham. (Pikhurko has independently disproved this conjecture by a very different method.)

A central question in this area, known as Turán's problem, is to determine the Turán density of $K_{4}^{(3)}=\{123,124,134,234\}$. Turán conjectured that this should be 5/9. Razborov [On 3-hypergraphs with forbidden 4-vertex configurations in SIAM J. Disc. Math. 24 (2010), 946-963] showed that if we consider the induced Turán problem forbidding $K_{4}^{(3)}$ and $E_{1}$, the 3 -graph with 4 vertices and a single edge, then the Turán density is indeed $5 / 9$. We give some new non-induced results of a similar nature, in particular we show that $\pi\left(K_{4}^{(3)}, H\right)=5 / 9$ for a 3-graph $H$ satisfying $\pi(H)=3 / 4$.

We end with a number of open questions focusing mainly on the topic of which values can occur as Turán densities.

Our work is mainly computational, making use of Razborov's flag algebra framework. However all proofs are exact in the sense that they can be verified without the use of any floating point operations. Indeed all verifying computations use only integer operations, working either over $\mathbb{Q}$ or in the case of irrational Turán densities over an appropriate quadratic extension of $\mathbb{Q}$.
\end{abstract}

*This author is a Royal Society University Research Fellow 


\section{Introduction}

An $r$-graph is a pair $F=(V(F), E(F))$ where $V(F)$ is a set of vertices and $E(F)$ is a family of $r$-subsets of $V(F)$ called edges. So a 2-graph is a simple graph. For ease of notation we usually identify an $r$-graph with its edge set. The number of edges in $F$ is denoted by $e(F)$.

Given a family of $r$-graphs $\mathcal{F}$ we say that an $r$-graph $H$ is $\mathcal{F}$-free if $H$ does not contain a subgraph isomorphic to any member of $\mathcal{F}$. For any integer $n \geqslant 1$ we define the Turán number of $\mathcal{F}$ to be

$$
\operatorname{ex}(n, \mathcal{F})=\max \{e(H): H \text { is } \mathcal{F} \text {-free, }|V(H)|=n\} .
$$

Even in the simplest case of 2-graphs this parameter can be very difficult to determine exactly thus we will consider the related asymptotic density.

The Turán density of $\mathcal{F}$ is defined to be the following limit (a simple averaging argument due to Katona, Nemetz and Simonovits [19] shows that it always exists)

$$
\pi(\mathcal{F})=\lim _{n \rightarrow \infty} \frac{\operatorname{ex}(n, \mathcal{F})}{\left(\begin{array}{l}
n \\
r
\end{array}\right)} .
$$

There are two general questions that are of interest to us.

Question 1. Given a family of $r$-graphs $\mathcal{F}$, what is $\pi(\mathcal{F})$ ?

Question 2. Which values in [0,1) are Turán densities of families of $r$-graphs?

For $r=2$ the Erdős-Stone-Simonovits theorem answers both questions completely.

Theorem 3 (Erdös and Stone [13], Erdős and Simonovits [12]). Let $\mathcal{F}$ be a family of 2-graphs. If $t=\min \{\chi(F): F \in \mathcal{F}\} \geqslant 2$ then

$$
\pi(\mathcal{F})=1-\frac{1}{t-1} .
$$

In particular the set of Turán densities of 2-graphs is $\{0,1 / 2,2 / 3,3 / 4, \ldots\}$.

For $r \geqslant 3$ remarkably little is known. One general result for $r$-graphs is the following theorem of Erdös. An $r$-graph is $r$-partite if its vertices can be partitioned into $r$ classes so that each edge meets each class exactly once.

Theorem 4 (Erdös [11]). If $K^{(r)}(t)$ is the complete $r$-partite $r$-graph with $t$ vertices in each class then $\pi\left(K^{(r)}(t)\right)=0$. 
Since $\lim _{t \rightarrow \infty} e\left(K^{(r)}(t)\right) /\left(\begin{array}{c}t r \\ r\end{array}\right)=r ! / r^{r}$ and all subgraphs of $K^{(r)}(t)$ are $r$-partite we have the following simple corollary.

Corollary 5. If $\mathcal{F}$ is a family of $r$-graphs then either at least one member of $\mathcal{F}$ is $r$-partite and so $\pi(\mathcal{F})=0$, or none are $r$-partite and $\pi(\mathcal{F}) \geqslant r ! / r^{r}$.

Essentially the only other general result is the following.

Theorem 6 (Mubayi [21] and Pikhurko [23]). For $3 \leqslant r \leqslant t$ let $H_{t}^{r}$ be the $r$-graph with vertices $x_{i}$ for $1 \leqslant i \leqslant t$ and $y_{i j}^{k}$ for $1 \leqslant i<j \leqslant t, 1 \leqslant k \leqslant r-2$ together with edges $x_{i} x_{j} y_{i j}^{1} \cdots y_{i j}^{r-2}$, for $1 \leqslant i<j \leqslant t$.

$$
\pi\left(H_{t+1}^{r}\right)=\frac{r !}{t^{r}}\left(\begin{array}{l}
t \\
r
\end{array}\right)
$$

Attention has focused mainly on Question 1, in particular a lot of work has gone into determining or giving bounds for the Turán density of particularly simple 3-graphs such as $K_{4}^{-}=\{123,124,134\}, K_{4}^{(3)}=\{123,124,134,234\}$ and $F_{3,2}=\{123,145,245,345\}$.

In Section 2 we give some new Turán results for individual 3-graphs. In particular we give the first examples of single 3-graphs with Turán density 5/9 for which Turán's construction $T_{n}$ is asymptotically extremal (see Section 2.3 for definitions).

In Section 3 we focus on Question 2, in particular giving the first examples of irrational Turán densities of finite families of 3 -graphs.

We then return to the classical "Turán Problem" of determining $\pi\left(K_{4}^{(3)}\right)$.

Given an exact Turán density result for a family of $r$-graphs $\mathcal{F}$ there are two very natural questions one can ask. Firstly, what is the exact Turán number ex $(n, \mathcal{F})$ ? Secondly, is there a "stability" result saying that all almost extremal $\mathcal{F}$-free $r$-graphs have essentially the same structure? Pikhurkho [25] answered both of these questions in the case of $\mathcal{F}=\left\{K_{4}^{(3)}, E_{1}\right\}$, where $E_{1}$ is the 3 -graph with 4 vertices and a single edge. We are able to give stability versions of all of our results from Sections 2 and 4. Luckily once we have a "flag algebra proof" of the Turán density of each family we can prove stability without having to consider each family in turn. Essentially we prove one stability result for each construction, the details are given in Section 5 .

Stability probably also holds for the results in Section 3 but we have not proved this. The question of determining the exact Turán number for each of the families we consider seems much more difficult although it is plausible that for all of our $\operatorname{results} \operatorname{ex}(n, \mathcal{F})$ is given by the corresponding construction for all sufficiently large $n$.

All discussion of the computational proofs is deferred to the final section, with transcripts of the actual proofs forming a separate appendix. However we emphasise that all of our proofs can be verified using only integer operations and hence are genuine proofs rather than numerical results with the potential for rounding errors. These proofs are set in 
Razborov's flag algebra framework [26] and make heavy use of semi-definite programming (see [5] and [27]).

A key tool we will make use of is the "blow-up" of an $r$-graph. Given an $r$-graph $F$ and an integer $t \geqslant 1$ the blow-up $F(t)$ is the 3 -graph formed by replacing each vertex of $F$ with a class of $t$ vertices and inserting a complete $r$-partite $r$-graph between any vertex classes corresponding to an edge in $F$. Given a family $\mathcal{F}=\left\{F_{1}, \ldots, F_{s}\right\}$ of $r$-graphs and an integer vector $\mathbf{t}=\left(t_{1}, \ldots, t_{s}\right)$ with each $t_{i} \geqslant 1$, we define the $\mathbf{t}$-blow-up of $\mathcal{F}$ to be $\mathcal{F}(\mathbf{t})=\left\{F_{i}\left(t_{i}\right): 1 \leqslant i \leqslant s\right\}$.

The following result will be extremely useful.

Theorem 7 (Brown and Simonovits [8]). If $\mathcal{F}=\left\{F_{1}, \ldots, F_{s}\right\}$ is a family of $r$-graphs and $\mathbf{t}=\left(t_{1}, \ldots, t_{s}\right)$ is an integer vector with each $t_{i} \geqslant 1$ then $\pi(\mathcal{F}(\mathbf{t}))=\pi(\mathcal{F})$.

In particular we have the following corollary that will often simplify the computations we perform. We will write $F \leqslant G$ to mean " $F$ is contained in a blow-up of $G$ ".

Corollary 8. If $\mathcal{F}$ is a family of $r$-graphs and $G_{1}, G_{2}$ are r-graphs with $G_{1} \leqslant G_{2}$ then

(i) $\pi\left(\mathcal{F} \cup G_{1}\right) \leqslant \pi\left(\mathcal{F} \cup G_{2}\right)$,

(ii) $\pi\left(\mathcal{F} \cup G_{1}\right)=\pi\left(\mathcal{F} \cup G_{1} \cup G_{2}\right)$.

Proof. Let $t \geqslant 1$ satisfy $G_{1} \subseteq G_{2}(t)$. Theorem 7 implies that $\pi\left(\mathcal{F} \cup G_{2}(t)\right)=\pi\left(\mathcal{F} \cup G_{2}\right)$. While $G_{1} \subseteq G_{2}(t)$ implies that $\pi\left(\mathcal{F} \cup G_{1}\right) \leqslant \pi\left(\mathcal{F} \cup G_{2}(t)\right)$. Hence (i) holds.

For (ii) we note that $\pi\left(\mathcal{F} \cup G_{1}\right) \geqslant \pi\left(\mathcal{F} \cup G_{1} \cup G_{2}\right)$ is trivial. While (i) implies that

$$
\pi\left(\mathcal{F} \cup G_{1}\right)=\pi\left(\mathcal{F} \cup G_{1} \cup G_{1}\right) \leqslant \pi\left(\mathcal{F} \cup G_{1} \cup G_{2}\right) .
$$

For a detailed description of how we apply Corollary 8 (to prove Theorem 12) see the discussion in Section 7. Essentially we apply part (ii) repeatedly: if $F \leqslant G$ for each $G$ in some family $\mathcal{G}$ then $\pi(F)=\pi(F \cup \mathcal{G})$. This often leads to much more tractable computational problems.

When investigating new Turán density results of $r$-graphs we have to be clear about what makes a result new. Consider the following situation: we have an $r$-graph $G$ whose Turán density is known together with a sequence of asymptotically extremal examples $\left\{G_{n}\right\}_{n=1}^{\infty}$ (i.e. $G_{n}$ is a $G$-free $r$-graph of order $n$ and $\lim _{n \rightarrow \infty} e\left(G_{n}\right) /\left(\begin{array}{l}n \\ r\end{array}\right)=\pi(G)$ ). Given a subgraph $F$ of $G$ we obviously know that $\pi(F) \leqslant \pi(G)$ and moreover if $G_{n}$ is $F$-free for all $n \geqslant 1$ then $\pi(F)=\pi(G)$. Corollary 8 sometimes allows us to deduce new Turán densities by checking for "containment in blow-ups". We will not be interested in results that are implied by known Turán density results by taking blow-ups and applying Corollary 8 . (See the remark following Theorem 11 for an example of such a result.) 
Note that checking if $F \leqslant G$ can be computationally difficult. For example suppose $r=2$ and $G=K_{3}$. Checking if a given graph $F$ satisfies $F \leqslant K_{3}$ is equivalent to determining whether $F$ is 3-colourable: a well known NP-complete problem.

An $r$-graph $F$ is said to be covering if every pair of vertices from $V(F)$ belongs to an edge in $F$. For example, complete $r$-graphs are covering. Covering $r$-graphs are easier to deal with when checking containment in blow-ups.

Lemma 9. If $F$ and $G$ are $r$-graphs and $F$ is covering then $F \leqslant G$ iff $F \subseteq G$.

Proof. If $F$ is a subgraph of $G(t)$ for some $t \geqslant 1$ then each vertex in $V(F)$ belongs to a different class in $G(t)$ (since there is an edge of $F$ containing any pair of vertices). Thus $F$ is a subgraph of $G$.

\section{Turán densities of individual 3-graphs}

We require a couple of basic definitions. For an integer $n \geqslant 1$ let $[n]=\{1,2, \ldots, n\}$. If $[n]=A_{1} \cup A_{2} \cup \cdots \cup A_{k}$ is a partition then we say that it is balanced if ||$A_{i}|-| A_{j}|| \leqslant 1$ for all $i, j \in[k]$.

\section{$2.1 \quad$ Density $2 / 9$}

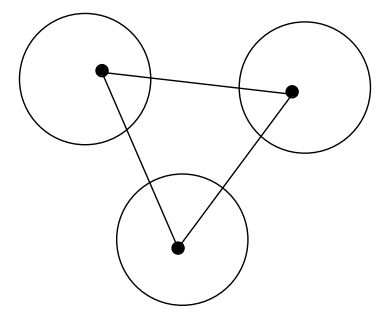

Figure 1: The balanced complete tripartite 3-graph, $S_{n}$.

Given a tripartition $[n]=V_{0} \cup V_{1} \cup V_{2}$ let $S\left(V_{0}, V_{1}, V_{2}\right)$ denote the complete tripartite 3 -graph with vertex classes $V_{0}, V_{1}, V_{2}$. Let $S_{n}$ denote the complete tripartite 3 -graph with the maximum number of edges (given by a balanced tripartition of $[n]$ ). Note that $e\left(S_{n}\right)=\left\lfloor\frac{n}{3}\right\rfloor\left\lfloor\frac{n+1}{3}\right\rfloor\left\lfloor\frac{n+2}{3}\right\rfloor$, and so $\lim _{n \rightarrow \infty} e\left(S_{n}\right) /\left(\begin{array}{l}n \\ 3\end{array}\right)=2 / 9$. Since all subgraphs of $S_{n}$ are tripartite this implies that any non-tripartite 3 -graph $F$ satisfies $\pi(F) \geqslant 2 / 9$.

The first Turán-type result for non-tripartite 3 -graphs was due to Bollobás [7]. Let $K_{4}^{-}=$ $\{123,124,134\}$ and $F_{5}=\{123,124,345\}$.

Theorem 10 (Bollobás [7]). If $\mathcal{F}=\left\{K_{4}^{-}, F_{5}\right\}$ then ex $(n, \mathcal{F})=e\left(S_{n}\right)$. In particular $\pi(\mathcal{F})=2 / 9$. 
This was followed by the Turán result for the single 3-graph $F_{5}$.

Theorem 11 (Frankl and Füredi [16]). If $n \geqslant 3000$ then ex $\left(n, F_{5}\right)=e\left(S_{n}\right)$. In particular $\pi\left(F_{5}\right)=2 / 9$.

In fact, as a Turán density result, Theorem 11 does not meet our definition of a new result since Corollary 8 (ii) allows us to deduce that $\pi\left(F_{5}\right)=2 / 9$ from Theorem 10: take $\mathcal{F}=\emptyset$, $G_{1}=F_{5}, G_{2}=K_{4}^{-}$and note that $F_{5} \subseteq K_{4}^{-}(2)$.

Theorem 6 tells us that we also have $\pi\left(H_{4}^{3}\right)=2 / 9$, however this is implied by $\pi\left(F_{5}\right)=2 / 9$ and Theorem 7 , since $H_{4}^{3} \subseteq F_{5}(3)$.

The following new result implies all of the aforementioned results.

Theorem 12. If $H=\{123,124,345,156\}$ then $\pi(H)=2 / 9$.

Note that $H$ is not contained in a blow-up of $F_{5}$ so this is a genuinely new result.

The proof of Theorem 12 uses Razborov's flag algebras framework [26], [27] as well as Corollary 8. It is a straightforward calculation in this setting. For a general discussion of our methods see Section 7 . A detailed computational proof can be found in the appendix file 2-9-prf.txt.

\subsection{Density $4 / 9$}

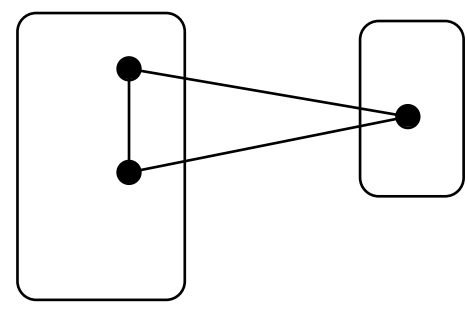

Figure 2: A $(2,1)$-colourable 3-graph.

Given a bipartition $[n]=V_{0} \cup V_{1}$ let $J\left(V_{0}, V_{1}\right)$ denote the 3 -graph with vertex set $[n]$ and edges consisting of all triples meeting $V_{0}$ in two vertices and $V_{1}$ in one vertex. We call this the complete $(2,1)$-colourable 3 -graph with classes $V_{0}$ and $V_{1}$. We say that a 3-graph $G$ is $(2,1)$-colourable if $G$ is isomorphic to a subgraph of $J\left(V_{0}, V_{1}\right)$ for some bipartition $[n]=V_{0} \cup V_{1}$. Let $J_{n}$ denote the $(2,1)$-colourable 3-graph of order $n$ with the maximum number of edges. A simple calculation shows that $J_{n}=J\left(V_{0}, V_{1}\right)$ for some bipartition with $\left|V_{0}\right|$ approximately twice as large as $\left|V_{1}\right|$ and so it is easy to check that $\lim _{n \rightarrow \infty} e\left(J_{n}\right) /\left(\begin{array}{l}n \\ 3\end{array}\right)=4 / 9$. Hence any 3 -graph $F$ that is not $(2,1)$-colourable satisfies $\pi(F) \geqslant 4 / 9$.

An example of a non- $(2,1)$-colourable 3 -graph is $F_{3,2}=\{123,145,245,345\}$. 
Theorem 13 (Füredi, Pikhurko and Simonovits [18]). For all $n \geqslant 3$ we have ex $\left(n, F_{3,2}\right)=$ $e\left(J_{n}\right)=\max _{k}(n-k)\left(\begin{array}{c}k \\ 2\end{array}\right)$. In particular $\pi\left(F_{3,2}\right)=4 / 9$.

We do not have an extension of this result, however we do have two new examples.

Theorem 14. The 3-graphs $G_{1}$ and $G_{2}$, given below, are non-(2,1)-colourable and satisfy $\pi\left(G_{1}\right)=\pi\left(G_{2}\right)=4 / 9$,

$$
G_{1}=\{123,124,134,235,245,156\}, \quad G_{2}=\{123,124,135,345,146,256\} .
$$

We note that the three examples of 3-graphs with Turán density 4/9: $F_{3,2}, G_{1}$ and $G_{2}$ are all incomparable under blow-ups. See Section 7 for discussion. Detailed computational proofs can be found in the appendix files 4-9-01-prf.txt and 4-9-02-prf.txt.

\subsection{Density $5 / 9$}

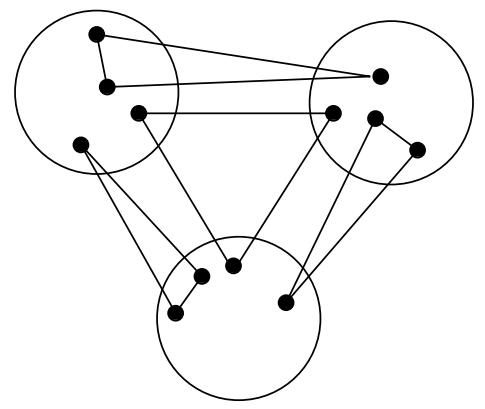

Figure 3: Turán's construction: $T_{n}$.

One obvious sequence of 3 -graphs with asymptotic density $5 / 9$ is given by taking the balanced blow-ups of $K_{6}^{(3)}$, the complete 3-graph of order 6. If $n$ is a multiple of six then $K_{6}^{(3)}(n / 6)$ has $20(n / 6)^{3}$ edges. This construction is extremal (at least asymptotically) for the 3-graph $H_{7}^{3}$ (see Theorem 6 ), and so $\pi\left(H_{7}^{3}\right)=5 / 9$.

Another sequence of 3-graphs with asymptotic density 5/9 was first introduced by Turán. Given a tripartition $[n]=V_{0} \cup V_{1} \cup V_{2}$ define the 3-graph $T\left(V_{0}, V_{1}, V_{2}\right)$ to have as edges all triples meeting each $V_{i}$ exactly once together with those triples containing two vertices from $V_{i}$ and one from $V_{i+1}$ (where subscripts are understood modulo 3 ). If the tripartition is balanced then we denote this 3 -graph by $T_{n}$ (again a simple calculation shows that $T_{n}$ has the maximum number of edges of all such 3-graphs).

Turán conjectured that $\operatorname{ex}\left(K_{4}^{(3)}, n\right)=e\left(T_{n}\right)$ and hence $\pi\left(K_{4}^{(3)}\right)=5 / 9$. This conjecture is still far from resolved and we will return to it in Section 4 . For now it is sufficient to note that previously there were no known examples of single 3 -graphs $F$ satisfying $\pi(F)=5 / 9$, with the lower bound provided by $T_{n}$. (Since $H_{t}^{3}$ is $(2,1)$-colourable for any $t$ we have $H_{t}^{3} \subseteq T_{n}$ for $n$ sufficiently large. In particular $H_{7}^{3} \subseteq T_{n}$.) 
Theorem 15. Each 3-graph $F$ in the list below satisfies $\pi(F)=5 / 9$ and $T_{n}$ is $F$-free. These 3-graphs are all incomparable with respect to blow-ups.

$$
\begin{aligned}
& \{123,124,134,125,245,136,346,156\}, \\
& \{123,124,134,125,135,245,345,236,456\}, \\
& \{123,124,134,125,135,245,126,236,146\}, \\
& \{123,124,134,125,135,345,126,236,246\}, \\
& \{123,124,134,125,235,345,126,246,156\}, \\
& \{123,124,134,125,235,136,346,156,356\}, \\
& \{123,124,134,125,135,245,126,136,346,456\}, \\
& \{123,124,134,125,135,345,126,236,146,156\}, \\
& \{123,124,134,125,135,245,126,236,346,356\}, \\
& \{123,124,134,125,135,345,126,236,346,356\}, \\
& \{123,124,134,125,135,146,246,156,256,456\}, \\
& \{123,124,134,125,135,146,246,156,356,456\} .
\end{aligned}
$$

See Section 7 for discussion of our proof methods, again we made extensive use of Corollary 8 (ii). Detailed computational proofs can be found in the appendix files 5-9-01-prf .txt to 5-9-12-prf.txt.

\subsection{Density $3 / 4$}

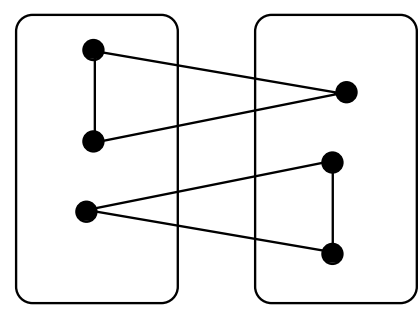

Figure 4: A bipartite 3-graph.

We say that a 3-graph is bipartite if there is a partition of its vertex set into two classes, neither of which contains an edge. Given a bipartition $[n]=V_{0} \cup V_{1}$ let $B\left(V_{0}, V_{1}\right)$ be the complete bipartite 3 -graph with vertex classes $V_{0}$ and $V_{1}$, i.e. its edges are all triples meeting both $V_{0}$ and $V_{1}$. If the bipartition is balanced then we denote this 3-graph by $B_{n}$. Clearly $B_{n}$ is a bipartite 3 -graph of order $n$ with the maximum number of edges. Moreover $\lim _{n \rightarrow \infty} e\left(B_{n}\right) /\left(\begin{array}{l}n \\ 3\end{array}\right)=3 / 4$ and so any non-bipartite 3 -graph $F$ satisfies $\pi(F) \geqslant 3 / 4$.

The first example of a 3-graph with Turán density $3 / 4$ was given by de Caen and Füredi [9], proving a conjecture of Sós. The Fano plane is the 3-graph $P G(2,2)=$ $\{123,145,356,167,257,347,246\}$. 
Theorem 16 (de Caen and Füredi [9]). The Fano Plane $P G(2,2)$ satisfies $\pi(P G(2,2))=$ $3 / 4$.

Their method was extended by Mubayi and Rödl [22] to show that a number of other 3 -graphs have Turán density $3 / 4$.

For $p, q \geqslant 1$ let $F_{p, q}$ be the 3-graph with vertex set $[p+q]$ and edges $\left(\begin{array}{c}{[p]} \\ 3\end{array}\right) \cup\{x y z: x \in$ $[p], y, z \in[p+q]-[p]\}$. Let $F_{3,3}^{\prime}$ be a copy of $F_{3,3}$ with two additional vertices, 7,8 , and four additional edges $178,278,478,578$. Let $F_{3,3}^{\prime \prime}$ be obtained from $F_{3,3}^{\prime}$ by adding two new vertices, $9, a$, and three edges, $19 a, 49 a, 79 a$. Let $F_{4,3}^{-}$be the 3 -graph obtained from $F_{4,3}$ by deleting the edge 156 . Let $F_{4,3}^{\prime-}$ be obtained from $F_{4,3}^{-}$by adding two vertices 8,9 , and adding three edges 289, 389, 589 .

Theorem 17 (Mubayi and Rödl [22]). Let $\mathcal{S}=\left\{F_{3,3}, F_{3,3}^{\prime}, F_{3,3}^{\prime \prime}, F_{4,3}^{-}, F_{4,3}^{\prime-}\right\}$. If $A, B \in \mathcal{S}$ and $A \subseteq F \subseteq B$ then $\pi(F)=3 / 4$.

We have the following new results.

Theorem 18. Each 3-graph $F$ in the list below satisfies $\pi(F)=3 / 4$ and $B_{n}$ is $F$-free. All of these 3-graphs are incomparable with respect to blow-ups.

$$
\begin{aligned}
& \{123,124,134,234,125,135,235,145,126,136,236,146,256,356\}, \\
& \{123,124,134,234,125,135,235,145,245,126,136,236,146,356,456\}, \\
& \{123,124,134,234,125,135,235,145,245,126,136,146,346,256,356,456\} .
\end{aligned}
$$

We remark that these are genuinely new results: the three 3-graphs in Theorem 18 are all covering and are not contained in any of the 3-graphs listed in Theorems 16 and 17 (thus by Lemma 9 we cannot deduce their Turán densities via blow-ups from the earlier results).

See Section 7 for discussion of our proof methods. Detailed computational proofs can be found in the appendix files 3-4-01-prf.txt to 3-4-03-prf.txt.

We note that for all of our new results (Theorems 12,14,15, 18) we have corresponding stability theorems (we defer a discussion of stability and exact Turán numbers to Section $5)$.

\section{New Turán densities for finite families}

In the previous section we focused almost exclusively on Turán densities of individual 3 -graphs. We now turn to the question of which values from $[0,1)$ can occur as Turán densities of families of $r$-graphs (Question 2). We will be interested in the size of the families in question and so require the following definitions. 
For $r \geqslant 3$ and $t \geqslant 1$ integers, define

$$
\begin{gathered}
\Pi_{t}^{(r)}=\{\pi(\mathcal{F}): \mathcal{F} \text { is a family of } r \text {-graphs and }|\mathcal{F}| \leqslant t\}, \\
\Pi_{\infty}^{(r)}=\{\pi(\mathcal{F}): \mathcal{F} \text { is a family } \mathcal{F} \text { of } r \text {-graphs }\}
\end{gathered}
$$

and

$$
\Pi_{\text {fin }}^{(r)}=\{\pi(\mathcal{F}): \mathcal{F} \text { is a finite family of } r \text {-graphs }\} .
$$

Obviously the following containments hold:

$$
\Pi_{1}^{(r)} \subseteq \Pi_{2}^{(r)} \subseteq \cdots \subseteq \Pi_{\mathrm{fin}}^{(r)} \subseteq \Pi_{\infty}^{(r)}
$$

To the best of our knowledge it is not known whether any of these containments are strict. The two general results we have regarding these sets of densities are Erdös's result for $r$-partite $r$-graphs (Theorem 4) and Mubayi and Pikhurko's result for $H_{t}^{r}$ (Theorem 6). Putting these together yields.

Corollary 19. For all $t \geqslant r \geqslant 2$

$$
\left[0, \frac{r !}{r^{r}}\right) \cap \Pi_{1}^{(r)}=\left[0, \frac{r !}{r^{r}}\right) \cap \Pi_{\infty}^{(r)}=\{0\}
$$

and

$$
\frac{r !}{t^{r}}\left(\begin{array}{l}
t \\
r
\end{array}\right) \in \Pi_{1}^{(r)}
$$

A useful tool, when searching for new Turán densities, is the Lagrangian of an $r$-graph. Let $F$ be an $r$-graph with vertex set $[n]=\{1,2, \ldots, n\}$. Define

$$
\Delta_{n}=\left\{\left(x_{1}, \ldots, x_{n}\right) \in \mathbb{R}^{n}: \sum_{i=1}^{n} x_{i}=1, x_{i} \geqslant 0\right\}
$$

For $x \in \Delta_{n}$ let

$$
\lambda(F, x)=r ! \sum_{\left\{i_{1}, i_{2}, \ldots, i_{r}\right\} \in F} \prod_{j=1}^{r} x_{i_{j}} .
$$

The Lagrangian of $F$ is $\lambda(F)=\max _{x \in \Delta_{n}} \lambda(F, x)$.

The Lagrangian of an $r$-graph is closely related to certain blow-ups of $F$ : it tells us how dense the densest blow-up of a subgraph of $F$ can be. We introduce a new set of densities:

$$
\Lambda^{(r)}=\{\lambda(F): F \text { is an } r \text {-graph }\} .
$$

Brown and Simonovits showed that the following containments hold. (Note that for $A \subseteq \mathbb{R}$ we denote the closure of $A$ by $\bar{A}$.) 
Theorem 20 (Brown and Simonovits [8]). If $r \geqslant 2$ then

$$
\Lambda^{(r)} \subseteq \Pi_{\infty}^{(r)}=\bar{\Pi}_{f i n}^{(r)}=\bar{\Lambda}^{(r)} .
$$

Thus in particular every Lagrangian of an $r$-graph is the Turán density of a corresponding infinite family of $r$-graphs. (In fact it is easy to see that for any $r$-graph $F$, the family $\mathcal{F}_{F}=\{G: G$ is an $r$-graph with $\lambda(G)>\lambda(F)\}$ satisfies $\pi\left(\mathcal{F}_{F}\right)=\lambda(F)$.)

For small 3-graphs it is straightforward to calculate the Lagrangian directly. We will use this to give some new examples of Turán densities of finite families of 3-graphs. In particular we give the first examples of irrational Turán densities for finite families, disproving the following conjecture of Chung and Graham [10].

Conjecture 21 (Chung and Graham [10] pg 95). If $\mathcal{F}$ is a finite family of $r$-graphs then $\pi(\mathcal{F})$ is rational.

Pikhurko [24] has also disproved this conjecture (for all $r \geqslant 3$ ). His proof is very different and the finite families he obtains are rather large.

We introduce the following notation: given an $r$-graph $G$ with vertex set $[k]$, a vector $x=\left(x_{1}, \ldots, x_{k}\right) \in \Delta_{k}$ and a large integer $n$; we define $G(x, n)$, the $n$ vertex blow-up of $G$ by $x$ to be the blow-up of $G$ in which vertex $i$ is replaced by a class of $\left\lfloor x_{i} n\right\rfloor$ vertices for $1 \leqslant i \leqslant k-1$ and vertex $k$ is replaced by a class of size $n-\sum_{i=1}^{k-1}\left\lfloor x_{i} n\right\rfloor$. (Thus if $\lambda(G)=\lambda(G, x)$ then $\left.\lim _{n \rightarrow \infty} e(G(x, n)) /\left(\begin{array}{l}n \\ r\end{array}\right)=\lambda(G).\right)$

Theorem 22. In each case below the finite family of 3-graphs $\mathcal{F}_{i}$ together with the 3-graph $G_{i}$ and weighting $x$ satisfy: $G_{i}(x, n)$ is $\mathcal{F}_{i}$-free for all $n \geqslant 1$ and $\pi\left(\mathcal{F}_{i}\right)=\lambda\left(G_{i}\right)$.

$$
\begin{aligned}
& G_{1}=\{123,124,125,345\}=F_{3,2} \\
& \lambda\left(G_{1}\right)=\frac{189+15 \sqrt{5}}{961}, \quad x_{1}=x_{2}=\frac{13+3 \sqrt{5}}{62}, x_{3}=x_{4}=x_{5}=\frac{6-\sqrt{5}}{31} . \\
& \mathcal{F}_{1}=\{\{123,124,135,146,156\},\{123,124,156,346,257\},\{123,124,156,347,567\}\} . \\
& G_{2}=\{123,234,345,145,125\}=C_{5}, \\
& \lambda\left(G_{2}\right)=\frac{6}{25}, \quad x_{1}=x_{2}=x_{3}=x_{4}=x_{5}=\frac{1}{5} . \\
& \mathcal{F}_{2}=\{\{123,124,134\},\{123,124,125,345\},\{123,124,135,256,167,467\}\} . \\
& G_{3}=\{123,124,134\}=K_{4}^{-} \\
& \lambda\left(G_{3}\right)=8 / 27, \quad x_{1}=\frac{1}{3}, x_{2}=x_{3}=x_{4}=\frac{2}{9} . \\
& \mathcal{F}_{3}=\{\{123,124,134,234\},\{123,124,125,345,346\},\{123,124,345,156,256\}, \\
& \quad \quad\{123,124,125,346,356,456\}\} .
\end{aligned}
$$




$$
\begin{aligned}
& G_{4}=\{123,124,125,134,135,145\}=F_{1,4} \\
& \lambda\left(G_{3}\right)=\frac{1}{3}, \quad x_{1}=\frac{1}{3}, x_{2}=x_{3}=x_{4}=x_{5}=\frac{1}{6} \text {. } \\
& \mathcal{F}_{4}=\{\{123,124,134,156,256\},\{123,124,134,125,126,357,367,457,467,567\}, \\
& \{123,124,345,156,257\}\} \\
& G_{5}=\{123,124,125,126,134,135,146,235,246,256,345,346,356,456\}=K_{6}^{(3)} \backslash C_{6}, \\
& \lambda\left(G_{5}\right)=\frac{7}{18}, \quad x_{1}=x_{2}=x_{3}=x_{4}=x_{5}=x_{6}=\frac{1}{6} \text {. } \\
& \mathcal{F}_{5}=\{\{123,124,135,145,346,256\},\{123,124,134,125,345,136,246\}, \\
& \{123,124,134,125,135,126,136,456\}\} \text {. } \\
& G_{6}=\{123,124,134,234,135,235,145,245\}=K_{5}^{(3)} \backslash\{125,345\} \\
& \lambda\left(G_{6}\right)=\frac{32}{81}, \quad x_{1}=x_{2}=x_{3}=x_{4}=\frac{2}{9}, x_{5}=\frac{1}{9} \text {. } \\
& \mathcal{F}_{6}=\{\{123,124,125,346,356,456\},\{123,124,135,256,346,456\},\{123,124,135,145 \text {, } \\
& 256,346\},\{123,124,134,125,126,356,456\},\{123,124,134,125,135,126,136,456\} \text {, } \\
& \{123,124,134,125,135,245,146,246,256\},\{123,124,134,125,135,345,126,146,346\} \text {, } \\
& \{123,124,134,125,135,235,245,146,246\}\} \text {. } \\
& G_{7}=\{123,124,134,234,125,135,235,145,245\}=K_{5}^{(3)} \backslash\{345\}, \\
& \lambda\left(G_{7}\right)=\frac{-35+13 \sqrt{13}}{27}, \quad x_{1}=x_{2}=\frac{5-\sqrt{13}}{6}, x_{3}=x_{4}=x_{5}=\frac{-2+\sqrt{13}}{9} . \\
& \mathcal{F}_{7}=\{\{123,124,135,345,146,256,346\},\{123,124,134,125,135,126,136,456\}, \\
& \{123,124,134,125,136,256,356,456\},\{123,124,134,125,135,145,126,136,146,156\} \text {, } \\
& \{123,124,134,234,125,135,245,236,146,346\}\} \text {. }
\end{aligned}
$$

In particular we have the following corollary.

Corollary 23. We have the following new Turán densities for finite families of 3-graphs

$$
\left\{\frac{189+15 \sqrt{5}}{961}, \frac{6}{25}, \frac{8}{27}, \frac{1}{3}, \frac{7}{18}, \frac{32}{81}, \frac{13 \sqrt{13}-35}{27}\right\} \subseteq \Pi_{\text {fin }}^{(3)}
$$

See Section 7 for discussion of our proof methods. Detailed computational proofs can be found in the appendix files Root5-prf.txt, 6-25-prf.txt, 8-27-prf.txt, 1-3-prf.txt, 7-18-prf.txt, 32-81-prf.txt and Root13-prf.txt. 


\section{Turán's problem}

Turán famously conjectured that $\operatorname{ex}\left(n, K_{4}^{(3)}\right)=e\left(T_{n}\right)$, thus in particular $\pi\left(K_{4}^{(3)}\right)=5 / 9$. If Turán's conjecture is true then there are in fact exponentially many non-isomorphic extremal examples of $K_{4}^{(3)}$ 3-graphs with ex $\left(n, K_{4}^{(3)}\right.$ ) edges (as described by Kostochka [20] and Fon-der-Flass [15]).

Much work has been done on Turán's conjecture and recently Razborov gave the upper bound of $\pi\left(K_{4}^{(3)}\right) \leqslant 0.561666$. Moreover, he noted that since Turán's construction $T_{n}$ contains no set of four vertices inducing a single edge it is natural to consider a related "induced Turán" problem.

Given a family of $r$-graphs $\mathcal{F}$ we say that an $r$-graph $G$ is induced $\mathcal{F}$-free if $G$ has no induced subgraph isomorphic to a member of $\mathcal{F}$. We then define the induced Turán number of a family of $r$-graphs $\mathcal{F}$ to be

$$
\operatorname{ex}_{\text {ind }}(n, \mathcal{F})=\max \{e(G): G \text { is an induced } \mathcal{F} \text {-free } r \text {-graph of order } n\} \text {. }
$$

The induced Turán density of $\mathcal{F}$ is then

$$
\pi_{\text {ind }}(\mathcal{F})=\lim _{n \rightarrow \infty} \frac{\operatorname{ex}_{\text {ind }}(n, \mathcal{F})}{\left(\begin{array}{l}
n \\
r
\end{array}\right)} .
$$

Theorem 24 (Razborov [27]). If $E_{1}$ is the 3-graph with 4 vertices and 1 edge then $\pi_{\text {ind }}\left(K_{4}^{(3)}, E_{1}\right)=5 / 9$.

Our aim in this section is to give some non-induced results of a similar nature. In particular we have an example of a 3-graph $H$ satisfying $\pi(H)=3 / 4$ and $\pi\left(K_{4}^{(3)}, H\right)=5 / 9$.

Theorem 25. Each of the 3-graphs $H_{i}$ listed below satisfies $\pi\left(K_{4}^{(3)}, H_{i}\right)=5 / 9$. They are all incomparable with respect to blow-ups. (For reference we also note the numerical upper bounds we found for $\pi\left(H_{i}\right)$ in each case).

$$
\begin{aligned}
& H_{1}=\{123,124,134,125,135,245,345,126,236,146,156,456\} \quad \pi\left(H_{1}\right)=3 / 4, \\
& H_{2}=\{123,124,134,125,135,126,236,146,346,356,456\} \quad \pi\left(H_{2}\right) \leqslant 0.613, \\
& H_{3}=\{123,124,134,125,135,245,345,126,236,346,356\} \quad \pi\left(H_{3}\right) \leqslant 0.613, \\
& H_{4}=\{123,124,134,125,135,245,236,146,346,156,456\} \quad \pi\left(H_{4}\right) \leqslant 0.608, \\
& H_{5}=\{123,124,134,125,135,245,345,236,146,256,456\} \quad \pi\left(H_{5}\right) \leqslant 0.608, \\
& H_{6}=\{123,124,134,125,135,245,345,126,136,246,346,456\} \quad \pi\left(H_{6}\right) \leqslant 0.597, \\
& H_{7}=\{123,124,134,125,345,136,246,256,356,456\} \quad \pi\left(H_{7}\right) \leqslant 0.595, \\
& H_{8}=\{123,124,134,125,135,236,146,246,156,256,456\} \quad \pi\left(H_{8}\right) \leqslant 0.594, \\
& H_{9}=\{123,124,134,125,135,245,236,346,356,456\} \quad \pi\left(H_{9}\right) \leqslant 0.555566, \\
& H_{10}=\{123,124,134,125,135,245,236,246,346,456\} \quad \pi\left(H_{10}\right) \leqslant 0.55555557 .
\end{aligned}
$$


The most interesting case of this result is $H_{1}$ so we focus on that now. It is straightforward to check that $H_{1}$ has a subgraph isomorphic to $F_{3,3}$ and hence is not bipartite. Moreover $H_{1}$ is a subgraph of the second example from Theorem 18, hence $\pi\left(H_{1}\right)=3 / 4$. The proof of the second part of this theorem is again computational and can be found in the appendix, although a few remarks are in place.

Our proof of Theorem 25 mimics that of Razborov's proof of Theorem 24. In particular the flag algebra computation we perform makes use only of information contained in the $\left\{K_{4}^{(3)}, H_{1}\right\}$-free 3 -graphs of order 6. (In Razborov's case these are replaced by the $K_{4}^{(3)}$ free 3 -graphs of order 6 with no induced $E_{1}$.) There are precisely 964 non-isomorphic $K_{4}^{(3)}$-free 3 -graphs of order 6 . Of these exactly 34 do not contain an induced $E_{1}$ and thus play a role in Razborov's proof of Theorem 24. However it turns out that 962 of the $K_{4}^{(3)}$-free 3 -graphs are $H_{1}$-free and are thus considered in the proof of Theorem 25 .

See the final section for discussion of our proof methods. Detailed computational proofs of the results in this section can be found in the appendix files $\mathrm{K} 4+\mathrm{H}-01$-prf.txt to K4+H-10-prf.txt.

A natural question to ask is whether any of the other $K_{4}^{(3)}$-free 3-graphs with $e\left(T_{n}\right)$ edges (described by Kostochka [20]) are also $H_{i}$-free for each $i$. Although some of the other constructions are $H_{i}$-free for some $i$ we have a stability result saying that all almost extremal examples of $\left\{K_{4}^{(3)}, H_{i}\right\}$-free 3-graphs have essentially the same structure as Turán's construction $T_{n}$. See the next section for details.

\section{$5 \quad$ Stability and exactness}

Given a family of $r$-graphs $\mathcal{F}$, we call a sequence of $r$-graphs $\left\{G_{n}\right\}_{n=1}^{\infty}$ almost extremal for $\mathcal{F}$ if each $G_{n}$ is $\mathcal{F}$-free of order $n$ with $d\left(G_{n}\right)=\pi(\mathcal{F})+o(1)$.

Theorem 26 (Stability). Let $\mathcal{F}$ be one of the families of 3-graphs whose Turán density is determined in Theorem 12, 14, 15, 18 or 25 and let $C_{n} \in\left\{S_{n}, J_{n}, T_{n}, B_{n}\right\}$ be the corresponding $\mathcal{F}$-free 3-graph with density $d\left(C_{n}\right)=\pi(\mathcal{F})+o(1)$. If $\left\{G_{n}\right\}_{n=1}^{\infty}$ is almost extremal for $\mathcal{F}$ then we can make $G_{n}$ isomorphic to $C_{n}$ by changing at most o $\left(n^{3}\right)$ edges.

It turns out that some cases of Theorem 26 require a little more work to prove than others. We give the proof in the "easy" case and then indicate how the other cases can be proved.

Our proof follows a similar argument to that given for the family $\mathcal{F}=\left\{K_{4}^{(3)}, E_{1}\right\}$ by Pikhurko (Theorem $2[25]$ ), although fortunately we can use the fact that each of the constructions we consider is characterised by its small induced subgraphs to avoid proving a separate result for each family (see Lemma 27).

If $G$ is an $r$-graph let $\mathcal{I}_{k}(G)=\{G[A]: A \subseteq V(G),|A|=k\}$ be the set of all $k$-vertex induced subgraphs of $G$. Given another $r$-graph $H$ we define $p(H ; G)$ to be the induced density of $H$ in $G$ : this is the probability that if $A \subseteq V(G)$ is a set of $|V(H)|$ vertices chosen uniformly at random then the subgraph induced by $A$ is isomorphic to $H$. 
Let $\mathcal{H}_{k}(\mathcal{F})$ be the family of all $\mathcal{F}$-free 3 -graphs of order $k$ up to isomorphism. We say that $H \in \mathcal{H}_{k}(\mathcal{F})$ is $\mathcal{F}$-sharp if there exists an almost extremal sequence $\left\{G_{n}\right\}_{n=1}^{\infty}$ for $\mathcal{F}$ such that $p\left(H ; G_{n}\right) \neq o(1)$. If $H \in \mathcal{H}_{k}(\mathcal{F})$ is not $\mathcal{F}$-sharp we say it is $\mathcal{F}$-negligible. We denote the family of $\mathcal{F}$-sharp 3-graphs of order $k$ by $\mathcal{H}_{k}^{\#}(\mathcal{F})$.

To motivate our next result consider the following trivial fact. If $G$ is a 2-graph of order at least 3 , with the property that all induced subgraphs of $G$ of order 3 are complete bipartite graphs then $G$ itself is a complete bipartite graph. In fact analogous results hold for the 3-graph properties we are interested in.

We say that an $r$-graph property $\mathcal{P}$ is $k$-induced if for any $r$-graph $G$ of order at least $k$, $\mathcal{I}_{k}(G) \subseteq \mathcal{P} \Longrightarrow G \in \mathcal{P}$.

Lemma 27. The following 3-graph properties are all 6-induced

$$
\begin{aligned}
& \mathcal{P}_{S}=\{G: G \text { is a complete tripartite 3-graph }\}, \\
& \mathcal{P}_{J}=\{G: G \text { is a complete }(2,1) \text {-colourable 3-graph }\}, \\
& \mathcal{P}_{B}=\{G: G \text { is a complete bipartite 3-graph }\} .
\end{aligned}
$$

Proof of Theorem 26: Let $\mathcal{F}$ be one of the families given in the statement of Theorem 26 with corresponding extremal construction $C_{n} \in\left\{S_{n}, J_{n}, T_{n}, B_{n}\right\}$. Suppose that $\left\{G_{n}\right\}_{n=1}^{\infty}$ is almost extremal for $\mathcal{F}$.

Our flag algebra proof of the Turán density of $\mathcal{F}$ using 6-vertex 3-graphs also provides us with information about $\mathcal{H}_{6}^{\#}(\mathcal{F})$. The associated proof file, e.g. 5-9-05-prf .txt, contains a list of all 6-vertex 3 -graphs that potentially belong to $\mathcal{H}_{6}^{\#}(\mathcal{F})$. In the easy case (which we now assume we are in) this tells us that $\mathcal{H}_{6}^{\#}(\mathcal{F}) \subseteq \mathcal{I}_{6}\left(C_{n}\right)$, i.e. the only induced 6vertex subgraphs that can occur with positive induced density in $G_{n}$ are those that are found in the corresponding construction $C_{n}$.

If $\mathcal{F}$ is a family whose Turán density is 5/9 then Pikhurko's stability theorem (Theorem 2 [23]) for $\left\{K_{4}^{(3)}, E_{1}\right\}$ in fact also applies to $\mathcal{F}$, so let us suppose we have a family $\mathcal{F}$ whose Turán density is $2 / 9,4 / 9$ or $3 / 4$, determined in Theorem 12, 14, or 18 .

We can now apply the hypergraph removal lemma of Rödl and Schacht [28] and obtain a new sequence of 3-graphs $\left\{G_{n}^{\prime}\right\}_{n=1}^{\infty}$ satisfying $\mathcal{I}_{6}\left(G_{n}^{\prime}\right) \subseteq \mathcal{I}_{6}\left(C_{n}\right)$ by changing $o\left(n^{3}\right)$ edges. Thus, by Lemma 27, we know that $G_{n}^{\prime}$ is isomorphic to $C\left(V_{0}, V_{1}, V_{2}\right)$ for some partition $[n]=V_{0} \cup V_{1} \cup V_{2}$. The result then follows by elementary calculus since $e\left(C\left(V_{0}, V_{1}, V_{2}\right)\right)=$ $e\left(G_{n}^{\prime}\right)=e\left(C_{n}\right)+o\left(n^{3}\right)$ implies that the partition $V_{0}, V_{1}, V_{2}$ must be approximately that giving $C_{n}$ and hence by changing at most $o\left(n^{3}\right)$ edges in $G_{n}^{\prime}$ we can obtain $C_{n}$.

This completes the proof in the easy case when our flag algebra proof tells us that $\mathcal{H}_{6}^{\#}(\mathcal{F}) \subseteq \mathcal{I}_{6}\left(C_{n}\right)$. (This applies to the families 3-4-02, 5-9-05, 5-9-07, 5-9-08, 5-9-09, 5-9-10, 5-9-12, K4+H-01, K4+H+06.)

There are two slightly more complicated cases:

(1) Rather than determining $\pi(\mathcal{F})$ directly we made use of blow-ups and Corollary 8 . 
(This applies to the families 2-9, 4-9-01, 4-9-02, 5-9-01, 5-9-02, 5-9-03, $5-9-04,5-9-06,5-9-11$.

(2) Our flag algebra proof does not immediately give $\mathcal{H}_{6}^{\#}(\mathcal{F}) \subseteq \mathcal{I}_{6}\left(C_{n}\right)$. (This applies to the families $3-4-01,3-4-03,5-9-01,5-9-06, \mathrm{~K} 4+\mathrm{H}-02, \mathrm{~K} 4+\mathrm{H}+03, \mathrm{~K} 4+\mathrm{H}-04$, $\mathrm{K} 4+\mathrm{H}+05, \mathrm{~K} 4+\mathrm{H}-07, \mathrm{~K} 4+\mathrm{H}+08, \mathrm{~K} 4+\mathrm{H}-09, \mathrm{~K} 4+\mathrm{H}+10$.)

We can deal with (1) as follows. If we used Corollary 8 to determine $\pi(\mathcal{F})$ then we have an auxillary family $\mathcal{F}^{\prime}$ and a flag algebra proof determining $\pi\left(\mathcal{F}^{\prime}\right)$. Moreover for each $F^{\prime} \in \mathcal{F}^{\prime}$ there exists $F \in \mathcal{F}$ such that $F \leqslant F^{\prime}$. Now suppose that $\left\{G_{n}\right\}_{n=1}^{\infty}$ is an almost extremal sequence for $\mathcal{F}$. We need to show that by changing at most $o\left(n^{3}\right)$ edges in $G_{n}$ we can obtain a sequence of 3-graphs $\left\{G_{n}^{\prime}\right\}_{n=1}^{\infty}$ that is almost extremal for $\mathcal{F}^{\prime}$ (this will return us to the easy case of the proof). We can do this using the hypergraph removal lemma as long as we know that all 6 vertex 3 -graphs $H$ that are not $\mathcal{F}^{\prime}$-free satisfy $p\left(H ; G_{n}\right)=o(1)$. This is straightforward to prove. Suppose there exists a 6 vertex 3 -graph $H$ such that $p\left(H ; G_{n}\right) \neq o(1)$ and $H$ is not $\mathcal{F}^{\prime}$-free. Then there exist $F \in \mathcal{F}, F^{\prime} \in \mathcal{F}^{\prime}, t \geqslant 1$ such that $F^{\prime}$ is a subgraph of $H$ and $F$ is a subgraph of $F^{\prime}(t)$. Moreover there exists $\epsilon>0$ and a subsequence $\left\{G_{n_{k}}\right\}_{k=1}^{\infty}$ such that $p\left(H ; G_{n_{k}}\right) \geqslant \epsilon$ for all $k$. Since $H$ contains $F^{\prime}$, a standard "supersaturation" argument implies that $G_{n_{k}}$ contains arbitrarily large blow-ups of $F^{\prime}$. Hence for $k$ large, $G_{n_{k}}$ contains $F^{\prime}(t)$ and so is not $\mathcal{F}$-free, a contradiction.

To deal with complication $(2)$ we have to show that the extra potentially $\mathcal{F}$-sharp 3 -graphs given by our flag algebra proof are in fact $\mathcal{F}$-negligible. We omit the the details of this argument since it is tedious but not difficult. (In fact there are only two subcases to deal with: the spurious $\mathcal{F}$-sharp graphs are identical in the cases of 3-4-01 and 3-4-03 and are also identical for the remaining families.)

If $G$ is a 3-graph we say that $a, b \in V(G)$ are twins if for all $x, y \in V \backslash\{a, b\}$ we have axy $\in G$ iff bxy $\in G$.

Proof of Lemma 2\%. In our arguments below we will be repeatedly examining small induced subgraphs of a given 3-graph $G$. If $a_{1}, \ldots, a_{k} \in V(G)$ then $G\left[a_{1} a_{2} \cdots a_{k}\right]$ is the subgraph induced by $\left\{a_{1}, \ldots, a_{k}\right\}$. Note that the vertices need not be distinct and so the induced subgraph may have less than $k$ vertices.

We first sketch the proof for $\mathcal{P}_{S}$. Let $G$ be a 3-graph of order $n \geqslant 6$ satisfying $\mathcal{I}_{6}(G) \subseteq \mathcal{P}_{S}$. Define a relation $\sim_{S}$ on $V=V(G)$ by $a \sim_{S} b$ iff $a=b$ or there exist distinct $c, d \in V \backslash\{a, b\}$ such that $G[a b c d]=S(a b, c, d)=\{a c d, b c d\}$, in which case we say $a \sim_{S} b$ via $c d$. We claim that this defines an equivalence relation on $V$. We need to check transitivity. Suppose $a \sim_{S} b$ via $u v$ and $b \sim_{S} c$ via $x y$, then without loss of generality $G[a b u v x y]=S(a b, u x, v y)$ so $G[a c x y]=S(a c, x, y)$ and $a \sim_{S} c$ as required.

Next we claim that if $a \sim_{S} b$ then $a$ and $b$ are twins. Let $x, y \in V \backslash\{a, b\}$ and suppose $a x y \in G$. If $a \sim_{S} b$ via $c d$, then wlog $G[a b c d x y]=S(a b, c x, d y)$ so $b x y \in G$. Similarly if $b x y \in G$ then $a x y \in G$, so $a$ and $b$ are twins. 
If $G$ has no edges then $G=S([n], \emptyset, \emptyset)$ so suppose $x y z \in G$. It is easy to check that $x, y, z$ are all in different equivalence classes, say $V_{x}, V_{y}, V_{z}$. Moreover if $v \in V \backslash\{x, y, z\}$ then examining $G[v x y z]$ we see that $v \in V_{x} \cup V_{y} \cup V_{z}$. Finally, using the fact that related vertices are twins, we obtain $G=S\left(V_{x}, V_{y}, V_{z}\right)$.

For $\mathcal{P}_{J}$ the proof is very similar, the main difference being that we define $a \sim_{J} b$ iff $a=b$ or there exist distinct $c, d \in V \backslash\{a, b\}$ such that $G[a b c d]=J(a b, c d)=\{a b c, a b d\}$. Again $\sim_{J}$ is an equivalence relation: if $a \sim_{J} b$ via $u v$ and $b \sim_{J} c$ via $x y$ but $a \chi_{J} c$ then wlog $c \neq u$ so $G[a b c u v x]=J(a b x, u v c)$ and $G[a b c u x y]=J(b c u, a x y)$, but acu is a non-edge in the former and an edge in the latter, a contradiction. We claim that if $a \sim_{J} b$ then $a$ and $b$ are twins. Let $x, y \in V \backslash\{a, b\}$ and suppose $a x y \in G$. If $a \sim_{J} b$ via $c d$ then by examining $G[a b c d x y]$ we see that $b x y \in G$. Similarly if $b x y \in G$ then $a x y \in G$, so $a$ and $b$ are twins.

Now either $G$ is empty, so $G=J(\emptyset, V)$, or $G$ contains an edge. Let $x y z \in G$ and suppose that no two distinct vertices are related. Let $v \in V \backslash\{x, y, z\}$, then wlog $G[x y z v]=$ $J(y z v, x)$. Now if $a, b \in V \backslash\{x\}$ are distinct then $G[x y z v a b]=J(y z v a b, x)$ so $a b x \in G$ and hence $G=J(V \backslash\{x\}, x)$.

Finally let $V_{x}$ be a largest equivalence class with $x, y \in V_{x}, x \neq y$. If $\Gamma_{x y}=\{z: x y z \in G\}$ then it is straightforward to check that $V(G)=V_{x} \cup \Gamma_{x y}$ is a partition of $V(G)$ into independent sets. Thus, since all vertices in $V_{x}$ are twins, we have $G=J\left(V_{x}, \Gamma_{x y}\right)$.

For $\mathcal{P}_{B}$ we define $a \sim_{B} b$ iff $a=b$ or there exist distinct $c, d, e \in V \backslash\{a, b\}$ such that $G[a b c d e]=B(a b, c d e)$ (so the only non-edge in $G[a b c d e]$ is $c d e$ ), in which case we say $a \sim_{B} b$ via cde. Again we claim $\sim_{B}$ is an equivalence relation. Suppose $a \sim_{B} b$ via $u v w$ and $b \sim_{B} c$ via $x y z$, but $a \chi_{B} c$. Without loss of generality we may suppose that $a \notin\{x, y\}, c \notin\{u, v\}$ and $\{x, y\} \neq\{u, v\}$. So $G[a b c u v w]=B(a b, c u v w)$ and $G[a b c x y z]=$ $B(b c, x y z a)$. This implies that $G[a b u v w x]=B(a b, u v w x)$ and $G[a b u v w y]=B(a b, u v w y)$. But then we have $G[a c u v x y]=B(a c, u v x y)$ so $a \sim_{B} c$. As before related vertices are twins: suppose $a \sim_{B} b$ via $c d e$. If $x, y \in V \backslash\{a, b\}$ and $a x y \in G$ but $b x y \notin G$ then $G[a b c d e]=B(a b, c d e)$ and $G[a b x y]=B(a, b x y)$. Now wlog $G[a b c d x y]=B(a c, b d x y)$, so $G[b c d e x y]=B(\emptyset, b c d e x y)$ is empty, a contradiction, since $b c d \in G$. Hence $a$ and $b$ are twins.

Let $V_{x}$ be a largest equivalence class. If $\left|V_{x}\right| \geqslant 2$ then suppose $x, y \in V_{x}$ are distinct. It is easy to check that $G=B\left(V_{x}, \Gamma_{x y}\right)$. If no equivalence class contains more than one vertex then either $G=B(\emptyset, V)$ is empty or one can check that there is a vertex $x$ such that $G=B(\{x\}, V \backslash\{x\})$.

We note that with a little extra work one can prove that all the 3-graph properties listed in Lemma 27 are in fact 5-induced. 


\section{$5.1 \quad$ Exactness}

Although we have stability for most of our results we have not proved any exact Turán numbers for the families we consider. In some, if not all cases, it may be possible to deduce an exact Turán number result from the stability theorem (along the same lines as Theorem 1 [25]) however the obvious approach to this would require a separate argument for each $\mathcal{F}$.

\section{Questions}

There are a number of obvious questions that arise from our work.

We were able to show a number of exact results for single 3-graphs with Turán density $2 / 9,4 / 9,5 / 9$ and $3 / 4$. Is there a systematic way to find or predict such results?

Question 28. Apart from blow-ups, are there any other operations under which Turán densities are invariant?

We were able to find a number of new Turán densities of finite families of 3-graphs by taking a small 3-graph $G$ and then investigating the Turán problem given by forbidding a family $\mathcal{F}_{G}$ of "small" 3-graphs not contained in any blow-up of $G$. In most cases we were able to show that $\pi\left(\mathcal{F}_{G}\right)=\lambda(G)$. Does this hold more generally?

Question 29. Is $\Lambda^{(r)} \subseteq \Pi_{\text {fin }}^{(r)}$ ?

Having given the first examples of finite families with irrational Turán densities we suspect there exist single $r$-graphs with irrational Turán densities (indeed the pentagon $C_{5}$ is quite possibly an example see [22], [27]).

Question 30. Do there exist single $r$-graphs with irrational Turán densities?

Another natural question is the following:

Question 31. For $r \geqslant 3$, which (if any) of the following containments between sets of densities are strict?

$$
\Pi_{1}^{(r)} \subseteq \Pi_{2}^{(r)} \subseteq \cdots \subseteq \Pi_{\mathrm{fin}}^{(r)} \subseteq \Pi_{\infty}^{(r)}
$$

Although we did not really consider "induced Turán problems" here, we could certainly ask analogous questions about the associated sets of densities for induced Turán problems.

\section{Computational proofs with flag algebras}

Our proofs make use of Razborov's flag algebra framework introduced in [26]. In particular we follow the method outlined by Razborov in [27]. For a precise description we refer back 
to Section 2 of our previous paper [5] where we provided a self-contained and detailed explanation of the method.

There are, however, two important ways in which the computations used to prove the results in this paper differ from our earlier work. Firstly we make extensive use of supersaturation, via Corollary 8. For example when computing the Turán density of $H=\{123,124,345,156\}$ (in Theorem 12) we use the fact that if

$$
H_{1}=\{123,124,134\}, \quad H_{2}=\{123,124,125,345\}, \quad H_{3}=\{123,124,135,245\}
$$

then $H \leqslant H_{i}$ for $i=1,2,3$.

Thus applying Corollary 8 (ii) we have $\pi(H)=\pi\left(\left\{H, H_{1}, H_{2}, H_{3}\right\}\right)$. This makes our computation significantly easier: there are 192 non-isomorphic 6 vertex 3-graphs that are $H$-free but only 38 of these are also $\left\{H_{1}, H_{2}, H_{3}\right\}$-free. (A very rough proxy for the difficulty of the computation is the final size of the proof file. In this case the use of Corollary 8 (ii) reduces our proof to less than $15 \%$ of the size of the smallest proof we could otherwise find. Moreover the computation completes in less than $10 \%$ of the time it would otherwise take.)

The second difference between the computations used to prove the results in this paper and those in [5] is that in this case we are proving exact sharp Turán density results. Razborov already achieved this for the induced $\left\{K_{4}^{(3)}, E_{1}\right\}$ problem (see Theorem 24) however in that case good use was made of the extremal construction to guide the conversion from numerical to exact result. We have found that even without using any information about the extremal construction we can often identify the sharp inequalities and zero eigenvalues, and hence make numerical results exact. In fact we have found that even for problems with many non-isomorphic extremal constructions we can sometimes prove exact Turán density results (see Baber [1] for an example of this in the hypercube). This is of particular interest since Turán's $K_{4}^{(3)}$ problem is a famous example where it is conjectured that there are many distinct extremal constructions [20], [15]. However we note that when proving the irrational Turán densities in Theorem 22 we made extensive use of the extremal constructions. For more discussion of the process used to produce our proofs from numerical results see Section 2.4 in [2]. Details of how to check and reprove our results are in the next subsection.

Note, that in order to achieve the required accuracy when converting floating point numerical results into exact proofs in $\mathbb{Q}$ (or indeed $\mathbb{Q}[\sqrt{5}], \mathbb{Q}[\sqrt{13}]$ for the irrational Turán densities) we make use of arbitrarily large integers. Indeed a glance at the proof file Root5-prf .txt reveals integers with over 150 digits.

\subsection{Source code}

Although all of our proof files are "human readable" their size precludes verification by hand. However (with the exception of the irrational results) they can all be verified 
using the program DensityChecker.cpp [3]. We also attach the source code used to generate the majority of our proofs: ExactDensityBounder.cpp [4]. This provides a simple command line program to give upper bounds for Turán densities of 3-graphs. It requires a semi-definite program solver: either csdp [6] or sdpa [17], both of which are freely available.

Detailed installation and usage instructions can be found in the source code files. We emphasise that these programs are both very easy to install and use.

\section{Acknowledgements}

We thank Oleg Pikhurko for drawing our attention to Conjecture 21 which he has also disproved by very different methods [24].

As we were preparing this paper we learnt of a similar project by Falgas-Ravry and Vaughan [14] to prove exact results for 3-graphs. Their approach is quite similar to ours although the actual results we have obtained are distinct. We have been able to independently verify all of the proofs of exact results announced in their paper and we attach these as proof files: FRV-12-49-prf.txt, FRV-5-18-prf.txt, FRV-3-4-prf.txt and FRV-3-8-prf.txt. (We note that the three results for which they obtain the exact Turán density 3/8, Theorems 6, 7 and 13 in [14], are in fact all implied by the single result $\pi\left(F_{3,2}, F_{1,5}\right)=3 / 8$, thus we only provide a single proof file in this case.)

We would like to thank a referee for very helpful comments and questions.

\section{References}

[1] R. Baber, Turán densities of hypercubes, arXiv:1201.3587

[2] R. Baber, Some Results in Extremal Combinatorics, PhD Thesis, UCL, 2011, http://www.ucl.ac.uk/ ucahjmt/Thesis.pdf

[3] R. Baber, DensityChecker.cpp, http://www.ucl.ac.uk/ ucahjmt/DensityChecker.cpp

[4] R. Baber, ExactDensityBounder.cpp, http://www.ucl.ac.uk/ ucahjmt/ExactDensityBounder.cpp

[5] R. Baber and J. Talbot, Hypergraphs do jump, Combin. Probab. Comput. $20161-$ 171, (2011).

[6] B. Borchers, CSDP, a C library for semi-definite programming, Optimisation Methods and Software 11 (1) 613-623, (1999).

[7] B. Bollobás, Three-graphs without two triples whose symmetric difference is contained in a third, Disc. Math. 8 21-24, (1974).

[8] W. G. Brown and M. Simonovits, Digraph extremal problems, hypergraph extremal functions, and the densities of graph structures, Disc. Math. 48 147-162 (1984). 
[9] D. de Caen and Z. Füredi, The maximum size of 3-uniform hypergraphs not containing a Fano Plane, J. Combin. Theory Ser. B. 78 274-276, (2000).

[10] F. Chung and R. Graham, Erdös on graphs: his legacy of unsolved problems, A.K. Peters, (1999).

[11] P. Erdős, On some extremal problems on r-graphs, Disc. Math. 1 1-6, (1971).

[12] P. Erdös and M. Simonovits, A limit theorem in graph theory, Studia Sci. Math. Hung. Acad. 1 51-57, (1966).

[13] P. Erdős and A.H. Stone, On the structure of linear graphs, Bull. Am. Math. Soc. 52 1087-1091, (1946).

[14] V. Falgas-Ravry and E. R. Vaughan, On applications of Razborov's flag algebra calculus to extremal 3-graph theory arXiv:1110.1623v1

[15] D. G. Fon-Der-Flass, A method for constructing $(3,4)$-graphs, Math. Zeitschrift 44 546-550, (1988).

[16] P. Frankl and Z. Füredi, A new generalization of the Erdös-Ko-Rado theorem, Combinatorica 3 341-349, (1983).

[17] K. Fujisawa, M. Kojima, K. Nakata, and M. Yamashita. SDPA (semidefinite programming algorithm) users manual - version 6.00. Technical Report B-308, Tokyo Institute of Technology, (1995).

http://sourceforge.net/projects/sdpa/files/sdpa-gmp/

[18] Z. Füredi, O. Pikhurko and M. Simonovits, On triple systems with independent neighbourhoods, Combin. Probab. Comput. 14 795-813, (2005).

[19] G. Katona, T. Nemetz and M. Simonovits, On a problem of Turán in the theory of graphs, Mat. Lapok 15, 228-238, (1964).

[20] A. Kostochka, A class of constructions for Turán's $(3,4)$ problem Combinatorica 2 187-192 (1982).

[21] D. Mubayi, A hypergraph extension of Turán's theorem, J. Combin. Theory Ser. B $96122-134$ (2006).

[22] D. Mubayi and V. Rödl, On the Turán number of triple systems, J. Combin. Theory Ser. A 100 136-152 (2002).

[23] O. Pikhurko, Exact computation of the hypergraph Turán function for expanded complete 2-graphs, accepted by J. Combin. Theory Ser. B, publication suspended indefinitely see http://www. math.cmu.edu/ pikhurko/Copyright.html

[24] O. Pikhurko, On Possible Turan Densities, submitted (2011).

[25] O. Pikhurko, The Minimum Size of 3-Graphs without a 4-Set Spanning No or Exactly Three Edges, Europ. J. Comb., 23 1142-1155 (2011).

[26] A. A. Razborov, Flag Algebras, Journal of Symbolic Logic, 72 (4) 1239-1282, (2007).

[27] A. A. Razborov, On 3-hypergraphs with forbidden 4-vertex configurations, SIAM J. Disc. Math. 24, (3) 946-963 (2010).

[28] V. Rödl and M. Schacht, Generalizations of the Removal Lemma, Combinatorica 29 467-501 (2009). 